\title{
LıBID: Reliable Identification of Obfuscated Third-Party Android Libraries
}

\author{
Jiexin Zhang \\ University of Cambridge \\ Cambridge, UK \\ jz448@cl.cam.ac.uk
}

\author{
Alastair R. Beresford \\ University of Cambridge \\ Cambridge, UK \\ arb33@cl.cam.ac.uk
}

\author{
Stephan A. Kollmann \\ University of Cambridge \\ Cambridge, UK \\ sak70@cl.cam.ac.uk
}

\begin{abstract}
Third-party libraries are vital components of Android apps, yet they can also introduce serious security threats and impede the accuracy and reliability of app analysis tasks, such as app clone detection. Several library detection approaches have been proposed to address these problems. However, we show these techniques are not robust against popular code obfuscators, such as ProGuard, which is now used in nearly half of all apps. We then present LiBID, a library detection tool that is more resilient to code shrinking and package modification than state-of-the-art tools. We show that the library identification problem can be formulated using binary integer programming models. LIBID is able to identify specific versions of third-party libraries in candidate apps through static analysis of app binaries coupled with a database of third-party libraries. We propose a novel approach to generate synthetic apps to tune the detection thresholds. Then, we use F-Droid apps as the ground truth to evaluate LIBID under different obfuscation settings, which shows that LIBID is more robust to code obfuscators than state-of-the-art tools. Finally, we demonstrate the utility of LiBID by detecting the use of a vulnerable version of the OkHttp library in nearly $10 \%$ of 3,958 most popular apps on the Google Play Store.
\end{abstract}

\section{CCS CONCEPTS}

- Security and privacy $\rightarrow$ Software reverse engineering.

\section{KEYWORDS}

Third-party library, Android, Obfuscation, ProGuard

\section{ACM Reference Format:}

Jiexin Zhang, Alastair R. Beresford, and Stephan A. Kollmann. 2019. LiBID: Reliable Identification of Obfuscated Third-Party Android Libraries. In Proceedings of the 28th ACM SIGSOFT International Symposium on Software Testing and Analysis (ISSTA '19), July 15-19, 2019, Beijing, China. ACM, New York, NY, USA, 12 pages. https://doi.org/10.1145/3293882.3330563

\section{INTRODUCTION}

In recent years, the smartphone ecosystem has developed rapidly, reaching around 1.4 billion by 2018 , with over $80 \%$ of the market share controlled by Android [32]. The success of the Android

Permission to make digital or hard copies of all or part of this work for personal or classroom use is granted without fee provided that copies are not made or distributed for profit or commercial advantage and that copies bear this notice and the full citation on the first page. Copyrights for components of this work owned by others than the author(s) must be honored. Abstracting with credit is permitted. To copy otherwise, or republish, to post on servers or to redistribute to lists, requires prior specific permission and/or a fee. Request permissions from permissions@acm.org.

ISSTA '19, fuly 15-19, 2019, Beijing, China

(c) 2019 Copyright held by the owner/author(s). Publication rights licensed to ACM ACM ISBN 978-1-4503-6224-5/19/07 ..\$15.00

https://doi.org/10.1145/3293882.3330563 ecosystem is fueled by the rich variety of third-party apps. Thirdparty libraries play a vital role in Android app development. They enable developers to: promote their apps with social media support; monetize their apps through advertisements; or simply facilitate app development and extend app functionality. In fact, a previous study has shown that, on average, over $60 \%$ of sub-packages in Android apps are from common libraries [33]. Nevertheless, the prevalence of third-party libraries can also bring new challenges that may affect the security and privacy of the Android ecosystem.

One challenge is that the presence of libraries may constitute a barrier for mobile app analysis. First, app clone detection schemes should not take library code into account during analysis. Since a large proportion of program code is contributed by libraries, which may well be used in many other apps without modification, the accuracy of detection schemes will be significantly skewed if library code is not properly excluded. Second, library code may result in significant overhead during static analysis. Static analysis is the basis for many Android research topics, but it is often challenged by computing power and resources [4]. Since libraries are often irrelevant to the main functionality of an app, removing them, or analyzing them separately, would improve efficiency [37].

Another major challenge is that third-party libraries may introduce serious security and privacy threats. One vulnerability in a popular library could compromise a great number of apps. For example, Facebook Android SDK version 3.15 [23] and certain versions of the OkHttp library [38], both of which were popular in Android apps, contained authentication vulnerabilities. In addition, several popular libraries have spied on SMS messages [24] or even established backdoors [25]. Attackers may also inject malicious code into popular libraries and redistribute them through unofficial platforms to remotely control smartphones or steal information. According to recent research, more than half of all Android apps with vulnerabilities were at risk due to libraries [36].

These challenges motivate the design of reliable and scalable techniques to identify libraries in mobile apps. However, there are several challenges. First, many apps adopt code obfuscators, such as ProGuard [16], to obfuscate the name of classes, fields, and methods; approaches based on simple identifier matching [28, 39] are not applicable for such apps. Second, many detection schemes tried to extract library candidates at scale by using clustering approaches [19, 21,33]. Although these schemes require no prior knowledge about the libraries before clustering, they require significant effort to label each cluster later. In addition, due to the lack of detailed information on libraries, clustering-based schemes cannot determine which version of a library is inside an app. Thus, they cannot be used to study whether apps use a vulnerable version 


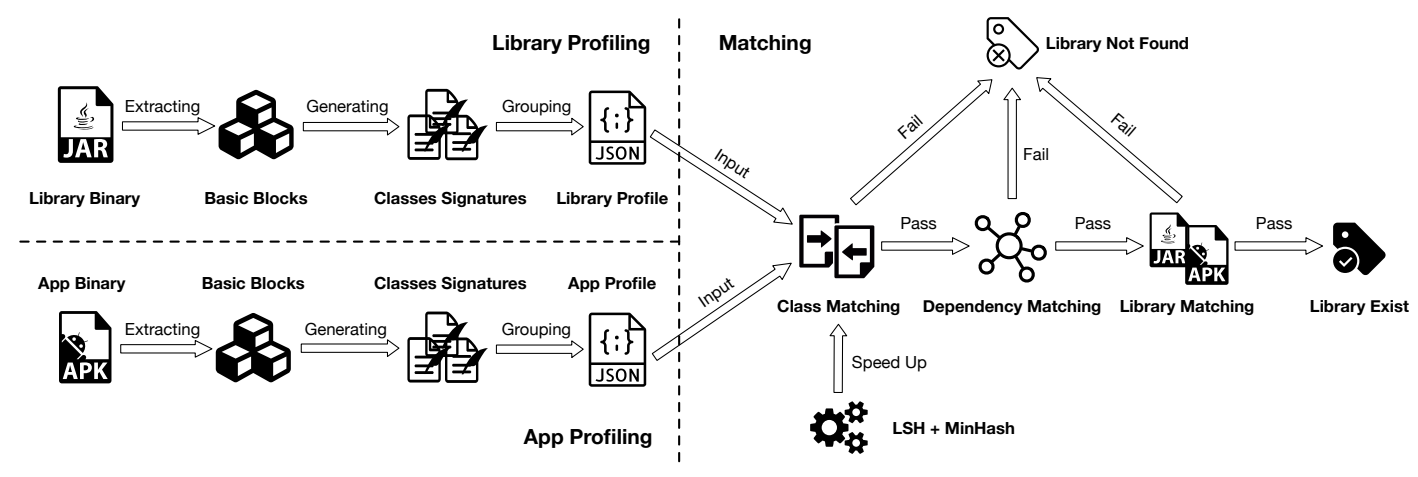

Figure 1: The workflow of LiBID

of libraries. Last, library code can be dynamically changed during the build process by code optimizers. For instance, ProGuard, an open source tool that has been integrated into the Android build system, will, by default, optimize all code and remove unused dead-code during the build process. This process is called code shrinking or dead-code elimination. In addition, ProGuard can apply package modification operations, which includes package flattening and class repackaging, to obfuscate packages and change class hierarchies. A recent study has shown that $49 \%$ of apps $(88 \%$ of obfuscated apps) used ProGuard and $21 \%$ of them applied package modification operations [34]. Most of the existing studies (e.g., LibRadar [21] and LibD [19]) failed to consider code shrinking and package modification operations, while others that did consider them (e.g., LibScout [5] and Orlis [35]) showed poor accuracy in our experiments.

Aim. We design a novel third-party Android library detection tool, LiBID, that can reliably identify the library version used in Android apps given the library and app binaries. LiBID is resilient to common code obfuscation techniques, including identifier renaming, code shrinking, control-flow randomization, and package modification.

Design. We start by building library and app profiles directly from their binaries by selecting obfuscation-resilient features. Then, we match library classes with app classes based on their profile. In particular, we formulate the constraints between matched classes and construct novel Binary Integer Programming (BIP) models to find the optimal match pairs that satisfy these constraints. LIBID also leverages a special Locality-Sensitive Hashing (LSH) technique to improve its efficiency and scalability by avoiding full pair-wise feature comparisons. In terms of computation complexity, we design two schemes for LIBID: LIBID-S and LiBID-A, with a focus on scalability and accuracy, respectively. In addition, we develop a systematic approach to generate synthetic apps with different versions of libraries. We use generated apps as the ground truth to tune the detection thresholds of LIBID and tentatively compare the results with state-of-the-art library detectors: LibScout, Orlis, and LibPecker [40]. Furthermore, we apply all library detectors to open source apps on F-Droid and benchmark their performance. Finally, we conduct a large-scale study on popular Google Play apps and identify a high proportion of popular apps using vulnerable libraries.
Contributions. We make the following contributions:

(1) We use recent software engineering methods, such as LSH ensemble, to design and implement a state-of-the-art thirdparty library detection system for Android.

(2) We are the first to formulate library matching constraints and convert library detection into a BIP problem.

(3) We propose a method of generating synthetic apps, where the ground truth is known, in order to empirically determine appropriate detection thresholds.

(4) We show LiBID achieves higher $F_{1}$ score than other state-ofthe-art methods both when no obfuscator is used and when ProGuard/Allatori/DashO is used.

(5) We show that LisID successfully detects vulnerable versions of the OKHttp library in nearly $10 \%$ of popular Google Play apps; LibScout only finds $7.5 \%$.

(6) We make all source code available for other researchers ${ }^{1}$.

\section{SYSTEM DESIGN}

The aim of LIBID is to reliably identify third-party libraries from Android binaries against popular obfuscation techniques, which requires fine-grained analysis that is usually time-consuming. To improve the usability of LIBID, we introduce two library identification schemes: LIBID-S (§3) and LIBID-A (§4), which focus on scalability and accuracy, respectively.

The workflow of LIBID is illustrated in Fig. 1. It consists of two major steps: profiling and matching. In particular, LIBID first generates a profile for every library and app binary. Then, these profiles are compared using a matching process. The output of the matching process allows LIBID to report the likelihood of any given library appearing in any given app. If there is a match, LIBID additionally reports the in-app package names of all matching libraries in an app, which enables researchers to quickly find the library code in the app.

\section{LIBID-S}

\subsection{Profiling}

As shown in Fig. 1, the profiling step can be divided into three stages. We do not differentiate the profiling of apps and libraries here because they use the same techniques. In particular, LIBID-S first

\footnotetext{
${ }^{1}$ https://github.com/ucam-cl-dtg/LibID
} 
Table 1: Grammar for the basic block signature

\begin{tabular}{c|c|c}
\hline \multicolumn{2}{c|}{ Instruction Type } & Abbreviation \\
\hline \multicolumn{2}{c}{ Basic Block } & $\mathrm{B}$ \\
\hline \multirow{2}{*}{ Field } & Read & $\mathrm{F} 0$ \\
\cline { 2 - 3 } & Write & $\mathrm{F} 1$ \\
\hline \multirow{2}{*}{ Method Call } & New Instance & M0 \\
\cline { 2 - 3 } & Other & M1 \\
\hline \multicolumn{2}{c|}{ String } & $\mathrm{S}$ \\
\hline \multicolumn{2}{c}{ If } & $\mathrm{I}$ \\
\hline \multicolumn{2}{c|}{ Return } & $\mathrm{R}$ \\
\hline \multicolumn{2}{c|}{ Goto } & $\mathrm{G}$ \\
\hline
\end{tabular}

constructs the Control Flow Graph (CFG) from a library binary and extracts all basic blocks from it. Then, LIBID-S builds a collection of basic block signatures that are invariant to identifier renaming, code shrinking, control flow randomization, and package modification techniques. The challenge is to do this while preserving as much information about the original code as possible. In this paper, we use the features and grammar presented in Table 1 to build a textual representation of the basic block signature, which records, in order, all field-related operations (read or write), method calls, the existence of strings inside each block, etc. If there are multiple field or method instructions in a basic block, there will be an abbreviation for each instruction in the signature. For example, if there are two field reads in a basic block, we will record two instances of $F 0$. The basic block signature also includes the name of called methods if they are from the Android SDK.

Furthermore, we associate the class features with each basic block signature by prefixing the basic block signature with four new components as shown in Fig. 2, which include:

- Class Access Flag: This field keeps the native Java access flag of the class since obfuscators typically do not change these class access flags.

- Superclass Name: If the class is extended from a class in the Android SDK, then this field will record the name of the superclass. Otherwise, this field is $[\mathrm{X}]$.

- Class Interfaces: This field only records class interfaces that are from the Android SDK. If there are multiple qualified interfaces, a separator | will be inserted in this field to separate them. This field is [] if the class does not implement any class from the Android SDK.

- Method Descriptor: We use the same format as LibScout [5] and OSSPolice [12] to profile class methods. Each method parameter is represented by its type in Dalvik and nonframework types will be replaced with a placeholder X.

This augmented basic block signature represents a feature of the class, and thus we call it a class signature; a class typically has a set of class signatures. An example of a class signature is given in Fig. 2. Then, each Android app or library can be characterized by a class signature dictionary, where the key is the name of its member class and the value is the set of class signatures of that class.

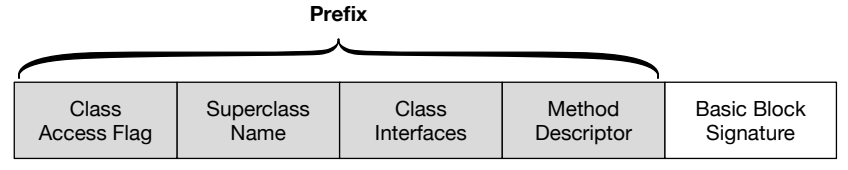

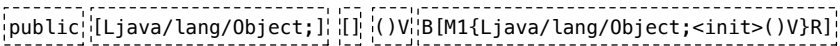

Figure 2: Format of a class signature with an example

\subsection{Matching}

After generating both app and library profiles, LIBID-S compares their similarity and calculates the confidence of the library being used in the app. We refer to this procedure as the matching process. As shown in Fig. 1, the matching process can be broken down into the following three steps: class matching, dependency matching and library matching.

Class Matching. This stage aims to find the candidate matches between library and app classes. An intuitive way to achieve our goal is to compare whether the signatures of these classes are the same. However, obfuscators can remove unused basic blocks from the original class or even delete the whole class, and thus a library class may have various signatures in different apps. In this regard, instead of looking for the same class signature sets, we define the class signature containment index as follows to quantify the similarity between two classes.

Definition 3.1 (Class Signature Containment). For two classes $c_{1}$ and $c_{2}$, whose signature set is $s_{1}$ and $s_{2}$, respectively, the class signature containment of $c_{1}$ in $c_{2}$ is defined as:

$$
S_{C}\left(c_{1}, c_{2}\right)=\frac{\left|s_{1} \cap s_{2}\right|}{\left|s_{1}\right|}
$$

In LiBID, library profiles are extracted directly from their binaries, and thus they contain a complete set of library class signatures. However, in-app versions of a library class may contain only a small subset of signatures due to the dead-code elimination. Therefore, LiBID-S only considers an app class $c_{a}$ as a candidate match to a library class $c_{l}$ if $S_{C}\left(c_{a}, c_{l}\right)$ is equal to 1 , since code shrinking does not change this index.

This containment index has been used to detect piggybacking apps $[11,13]$. However, it requires pair-wise comparison which is time-consuming. Locality-Sensitive Hashing (LSH) [2] provides an efficient solution to this problem. It pre-processes the dataset by creating signature hashes such that similar items have a higher chance of sharing the same hash. Here, LIBID applies a special LSH index technique named LSH Ensemble [42], which supports the containment query, to speed up the matching process.

Dependency Matching. The dependency matching stage aims to find the true match pairs from the candidates. Based on Definition 3.1, it is likely that a single class in an app is matched to multiple candidate library classes, or vice versa. However, this is problematic since each library class can produce at most one matching in-app class, and vice versa. For example, in Fig. 3, it is clear that L1 . class and L2. class cannot both be matched to A1. class. In fact, there can be at most two true matching pairs in Fig. 3. 


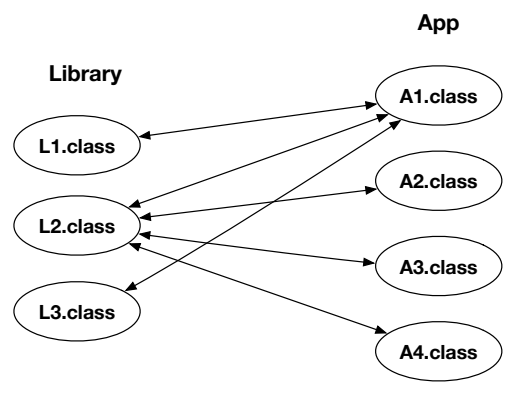

Figure 3: Class Matching result (ellipses stand for classes and the connections between them indicate a candidate match)

In general, suppose there are $m$ library classes $\mathrm{C}_{\mathrm{L}}$ that have candidate matches in the app and $n$ app classes $\mathrm{C}_{\mathrm{A}}$ that have candidate matches in the library. Then, we can generate a candidate match matrix $\mathbf{R} \in\{0,1\}^{m \times n}$, where $\mathbf{R}_{c_{l}, c_{a}}=1$ if and only if $c_{l} \in \mathbf{C}_{\mathbf{L}}$ and $c_{a} \in \mathrm{C}_{\mathrm{A}}$ are a candidate match. A true class match matrix $\mathrm{C} \in\{0,1\}^{m \times n}$ ensures a unique match between library and app classes should have the following uniqueness constraints:

$$
\begin{gathered}
\mathrm{C}_{c_{l}, c_{a}} \leq \mathrm{R}_{c_{l}, c_{a}}, \forall c_{l} \in \mathrm{C}_{\mathrm{L}}, c_{a} \in \mathrm{C}_{\mathrm{A}} \\
\sum_{c_{a} \in \mathrm{C}_{\mathrm{A}}} \mathrm{C}_{c_{l}, c_{a}} \leq 1, \quad \forall c_{l} \in \mathrm{C}_{\mathrm{L}} \\
\sum_{c_{l} \in \mathrm{C}_{\mathrm{L}}} \mathrm{C}_{c_{l}, c_{a}} \leq 1, \quad \forall a_{i} \in \mathrm{C}_{\mathrm{A}}
\end{gathered}
$$

Or in words, there should be at most one non-zero value in each row and column in $\mathbf{C}$ and $\mathbf{C}$ is a subset of $\mathbf{R}$.

In Android apps and libraries, each package may include a set of classes and several sub-packages. In most instances, obfuscators do not change the internal package structures. Therefore, LiBID-S by default will only match an app class with a library class if they are at the same level in the matching package. For example, in Fig. 4, suppose each library class is a candidate match to an app class. Here, we use the superscript * to denote candidate match pairs (e.g., a.class is a candidate match to $a^{*}$.class). If we want to check whether the library package Okio and app package a is a match, then the match pair (Okio/o.class, a/o*.class) is valid while (Okio/a.class, a/b/a*.class) is invalid because classes in the latter pair are at a different level to the matching package. However, if we are comparing package Okio with sub-package b in Fig. 4 (a), then that pair is valid because both a.class and $a^{*}$.class are directly under the matching package.

To make sure the matched class pairs are valid, we need some additional constraints. Suppose there are $m_{p}$ library packages $\mathbf{P}_{\mathbf{L}}$ and $n_{p}$ app packages $\mathbf{P}_{\mathbf{A}}$ that contain matched classes or their sub-packages contain matched classes. For example, if $\mathrm{C}_{\mathrm{L}}$ consists of two classes $(a / b / x . c l a s s$ and $a / c / y . c l a s s)$, then $P_{L}$ would have three elements $(a / b, a / c, a)$. Let $\mathbf{P} \in\{0,1\}^{m_{p} \times n_{p}}$ be the true package match matrix, then the hierarchy relation between classes can be ensured by the following hierarchy constraints:

$$
\begin{gathered}
\mathbf{P}_{p_{l}, p_{a}} \leq \mathbf{P}_{\text {parent }\left(p_{l}\right), \text { parent }\left(p_{a}\right)}, \forall p_{l} \in \mathbf{P}_{\mathbf{L}}, p_{a} \in \mathbf{P}_{\mathbf{A}} \\
\sum_{p_{a} \in \mathbf{P}_{\mathrm{A}}} \mathbf{P}_{p_{l}, p_{a}} \leq 1, \forall p_{l} \in \mathbf{P}_{\mathbf{L}}
\end{gathered}
$$

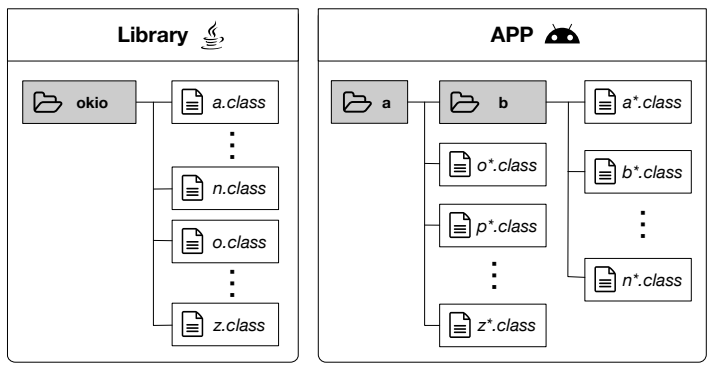

(a) Package flattening

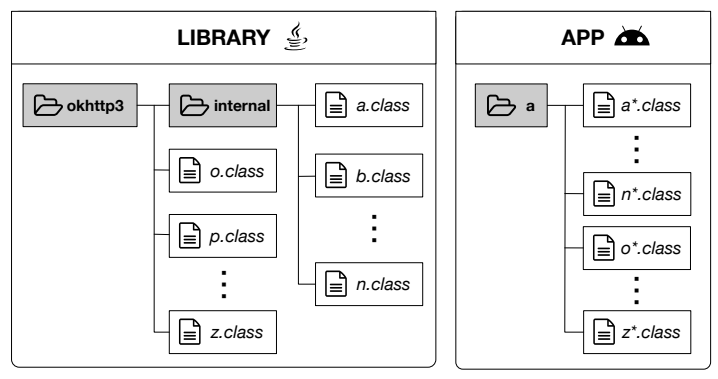

(b) class repackaging

Figure 4: Class hierarchy in app and library packages

$$
\begin{aligned}
& \sum_{p_{l} \in \mathbf{P}_{\mathbf{L}}} \mathbf{P}_{p_{l}, p_{a}} \leq 1, \forall p_{a} \in \mathbf{P}_{\mathrm{A}}
\end{aligned}
$$

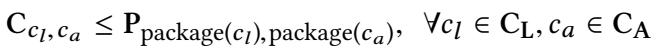

Constraint 3 a ensures the app and library packages can only be a match if their parent package also matches. In particular, if a matching package is already a top-level package (no parent package), this constraint will be ignored. Constraint $3 \mathrm{~b}$ and $3 \mathrm{c}$ make sure each library package can only be matched to at most one app package. Constraint $3 \mathrm{~d}$ ensures that classes can only be matched if their packages match. Collectively, these hierarchy constraints guarantee that matched class pairs have the same hierarchy.

The objective of LiBID-S is to find the maximum number of matched class pairs, which can be formulated to the following Binary Integer Programming (BIP) problem:

$$
\begin{array}{ll}
\operatorname{maximize} & \sum_{c_{l} \in \mathrm{C}_{\mathrm{L}}, c_{a} \in \mathrm{C}_{\mathrm{A}}} \mathrm{C}_{c_{l}, c_{a}} \\
\text { subject to } & \text { Constraint } 2 \text { and } 3
\end{array}
$$

The BIP problem has been extensively studied for many decades and there are many sophisticated software packages available to help solve this problem [30]. Previous work has shown that $21 \%$ of apps using ProGuard have applied package modification operations, which includes package flattening and class repackaging [34]. If package flattening is enabled, rule-specified packages will be put into a single parent package, but the structure of the package will be preserved. Notably, we do not require the hierarchy of packages to be the same. For instance, it is possible to have a match between package Okio and a/b in Fig. 4 (a). Therefore, LiBID-S is robust to package flattening and package renaming tools that could change the hierarchy of root packages but not internal package structures. 
By contrast, if class repackaging is applied, rule-specified classes will be moved to a single parent package, and thus the classes hierarchies in the original package will no longer be preserved. An example is given in Fig. 4 (b), where all classes in the library have been repackaged to package $a$ in the app. If we still use Constraint 3 , then many true class matches would be missed. To address this problem, we create a Class Repackaging Detection (CRD) mode. When LIBID-S runs in the CRD mode, every app package that does not have sub-packages could be the parent package of repackaged classes. Let $\mathbf{P}_{\mathrm{A}}^{\prime}$ be the collection of these app packages, then LiBID-S has the following hierarchy constraints when the CRD mode is on:

$$
\begin{gathered}
\sum_{p_{a} \in \mathbf{P}_{\mathrm{A}}^{\prime}} \mathbf{P}_{p_{l}, p_{a}} \leq 1, \quad \forall p_{l} \in \mathbf{P}_{\mathbf{L}} \\
\mathbf{P}_{p_{l}^{1}, p_{a}}=\mathbf{P}_{p_{l}^{2}, p_{a}}, \quad \forall p_{l}^{1}, p_{l}^{2} \in \mathbf{P}_{\mathrm{L}}, p_{a} \in \mathbf{P}_{\mathrm{A}}^{\prime} \\
\mathbf{C}_{c_{l}, c_{a}} \leq \mathbf{P}_{\text {package }\left(c_{l}\right), \operatorname{package}\left(c_{a}\right)}, \quad \forall c_{l} \in \mathrm{C}_{\mathrm{L}}, c_{a} \in \mathrm{C}_{\mathrm{A}}
\end{gathered}
$$

Or in words, all library packages could only be matched to a single app package that does not contain a sub-package. For example, in Fig. 4 (b), app package a could be a candidate match to the library package OkHttp3. In this way LIBID-S is also able to deal with class repackaging techniques if shrinking is not applied at the same time.

Library Matching. LIBID aims to pinpoint the third-party library version in Android apps. An intuitive solution is to check the proportion of library classes that are present in the app. However, some classes may have no viable signature, which we call unproductive classes. An example of an unproductive class is a class that contains only abstract methods (i.e., no basic block). Unproductive classes contribute little to the uniqueness of the library and will never be matched to other classes. Therefore, we exclude them from the proportion calculation. Nevertheless, this evaluation index is not robust against code shrinking. As a fix, we also consider the proportion of matched classes in the matched app package and define the library match index as follows:

Definition 3.2 (Library Match Index). Suppose the root package $P_{L}$ of library $L$ is matched to a package $P_{A}$ in app $A$, and there are $N_{C}$ true class match pairs between $L$ and $A$, then the library match index of $L$ in $A$ is:

$$
M(L, A)=\frac{N_{C}}{\min \left(N_{P_{L}}, N_{P_{A}}\right)}
$$

Where $N_{P_{L}}$ and $N_{P_{A}}$ are the number of productive classes in $P_{L}$ and $P_{A}$, respectively.

The library match index is suitable for quantifying the confidence of the presence of the library in the app. On the one hand, apps may use several libraries that share the same root package. In this case, $N_{C} / N_{P_{A}}$ can be pretty low even if the library is used by the app. On the other hand, if code shrinking is applied, then $N_{C} / N_{P_{L}}$ could be very low but $N_{C} / N_{P_{A}}$ would remain high. In both cases, $S_{C}(L, A)$ can still retain a high value. Therefore, we can decide whether app $A$ uses library $L$ using two thresholds:

$$
M(L, A)>\Gamma_{1} \text { and } \frac{N_{C}}{N_{P_{L}}}>\Gamma_{2}
$$

The threshold $\Gamma_{2}$ ensures there is enough information about the library for us to make a meaningful decision. In most cases, $M(L, A)$ should be close to $100 \%$ if app $A$ uses library L. Although LSH will inevitably introduce false negatives and false positives, the relation constraints can help to reduce false alarms during the matching process. However, if both class repackaging and shrinking are applied, then $M(L, A)$ can still be a small value. In this case, we can adjust the value of $\Gamma_{1}$ to balance between the detection of libraries and introducing false positives.

In general, each library update may modify only a small fraction of the code, and thus there may be multiple library versions that satisfy Constraint 5 . For each matched library, LiBID-S only keeps the versions that have the highest value of $M(L, A)$.

Summary. LiBID-S only relies on features that are consistent under code shrinking, control flow randomization, and package modification. The use of the LSH Ensemble greatly reduces the matching time by avoiding pair-wise comparison between classes and all calculations in LIBID-S are relatively light-weight. Therefore, LIBID-S is a suitable tool when computing resources are limited. Nevertheless, although we have considered the uniqueness and hierarchy constraints during the matching process, this coarsegrained analysis can still result in false matches between classes. In addition, if multiple libraries share the same root package in the app and each library only has a few classes remained in the app, LiBID-S may not be able to identify these libraries.

\section{LIBID-A}

\subsection{Profiling}

On the basis of LIBID-S, we design a more accurate library detection system named LIBID-A. LiBID-A employs finer-grained features (invocation, interface, and inheritance dependencies) during the library detection process, which enables it to not only find library matches for each app more precisely but also pinpoint class match pairs with higher accuracy. Overall, LiBID-S does not make use of the information about dependencies between different classes, which may result in incorrect matching. To address this problem, LIBID-A further records the following additional information:

- Method Calls: If a callee is obfuscatable, LiBID-A will record the name of the called class and method under the key of the caller class in the invocation dictionary.

- Class Inheritance: If a class's superclass is obfuscatable, LiBIDA will record the name of the superclass under the key of the current class in the inheritance dictionary.

- Class Interfaces: If the class's interface is obfuscatable, LiBIDA will record the name of the interface under the key of the current class in the interface dictionary.

Notably, we only keep records of the above information if the relating entities are obfuscatable (i.e., not from the Android SDK). Otherwise, they should have already been integrated into class signatures. Then, LiBID-A will build dependency graphs based on these dictionaries. Here, we define a dependency graph as an undirected graph, where each node corresponds to a class and each edge represents an invocation, inheritance or interface dependency between these two classes. Notably, these dictionaries are only used to construct the dependency graph. No static identifier (e.g., method and class name) will be utilized for string matching as they can easily be changed by identifier renaming. 


\subsection{Matching}

The matching process in LIBID-A also includes three major phases:

Class Matching. This process is the same as the class matching in LIBID-S. We use the class containment similarity index in Definition 3.1 to find all candidate class matches while applying $L S H$ Ensemble to speed up the matching process.

Dependency Matching. In contrast to LIBID-S, apart from uniqueness and hierarchy constraints, the dependency matching in LiBID-A also includes the following constraints:

- Invocation Constraints: For any two app classes $\left(c_{a}^{1}, c_{a}^{2} \in\right.$ $\mathrm{C}_{\mathrm{A}}$ ), if $c_{a}^{1}$ invokes a method in $c_{a}^{2}$ and the formatted method descriptor is $d$, and their true class match in the library is $c_{l}^{1}$ and $c_{l}^{2}$, respectively, then there must be a method call from $c_{l}^{1}$ to $c_{l}^{2}$ whose descriptor is also $d$. It is not necessarily true the other way around.

- Inheritance Constraints: For any two app classes $\left(c_{a}^{1}, c_{a}^{2} \in\right.$ $\mathrm{C}_{\mathrm{A}}$ ), if $c_{a}^{1}$ is the superclass of $c_{a}^{2}$, and their true class match in the library is $c_{l}^{1}$ and $c_{l}^{2}$, respectively, then $c_{l}^{1}$ must also be the superclass of $c_{l}^{2}$, and vice versa.

- Interface Constraints: For any two app classes $\left(c_{a}^{1}, c_{a}^{2} \in \mathrm{C}_{\mathrm{A}}\right)$, if $c_{a}^{2}$ is an interface of $c_{a}^{1}$, and their true class match in the library is $c_{l}^{1}$ and $c_{l}^{2}$, respectively, then there must be a method call from $c_{l}^{1}$ to $c_{l}^{2}$ whose descriptor is also $d$. It is not necessarily true the other way around.

The formulation of these constraints are very similar to LIBID-S, and thus we do not reiterate them here. Notably, the invocation and interface constraints do not work the other way around. The reason is that both method calls and interfaces can be deleted by code shrinking, but the superclass will always be kept if the child class exists. LIBID-A also has a CRD mode, which replaces the default hierarchy constraints with Constraint 4 while keeping other constraints the same.

The objective of LiBID-A can be formulated as follows:

$$
\text { maximize } \sum_{c_{l} \in \mathrm{C}_{\mathrm{L}}, c_{a} \in \mathrm{C}_{\mathrm{A}}} \mathrm{C}_{c_{l}, c_{a}}+w \sum_{c_{a}^{1}, c_{a}^{2} \in \mathrm{C}_{\mathrm{A}}}(\mathrm{V}+\mathrm{H}+\mathrm{T})_{c_{a}^{1}, c_{a}^{2}}
$$

subject to five types of constraints described in this section

where $\mathbf{V}, \mathrm{H}, \mathrm{T} \in\{0,1\}^{n \times n}$ are the invocation, inheritance, and interface matrix, respectively. Their element is equal to 1 if and only if there is corresponding dependency between $c_{a}^{1}$ and $c_{a}^{2} . w$ is a weighted parameter that should be small enough (e.g., 0.0001). In other words, LiBID-A aims to find the optimal solution that has the largest number of class match pairs. If there are multiple solutions, it selects the one with the largest number of dependency matches.

Library Matching. To reliably detect libraries when both class repackaging and shrinking are applied, LiBID-A utilizes the dependency graph to perform better library matching.

Relation Pruning: Although class repackaging can move classes from different packages into a single package, the dependency graphs stay the same. An example of the dependency graph of Okio 1.7.0 is shown in Fig. 6 in Appendix A. Therefore, we should only consider classes in the app package that are reachable from any matched classes. In other words, an app class needs to be in the same connected component with any matched class in the dependency graphs in order to be considered. We name this process relation pruning.

Ghost Hunting: Library classes may invoke methods from classes that exist in another library under the same package name. This is most common when libraries are developed by the same company. For instance, Leakcanary calls methods from Leakcanary-Watcher and they share the same root-package com/squareup/leakcanary. In this case, we cannot eliminate the interference of other libraries through relation pruning. Instead, for each library class, if it depends on another class that is not in the library and not from Android SDK, we will record this connection and refer to the connected class as a ghost class. For each ghost class, we find its matched app class by dependency matching and remove it from the dependency graphs. This process is named ghost hunting.

For each library and app match, we first implement ghost hunting to eliminate the distraction of ghost classes and then apply relation pruning to avoid the interference of classes that are not from the library but in the same app package. Let $N_{P_{A}}^{C}$ be the number of qualified app classes, then the definition of library match index for LIBID-A can be updated as:

$$
M(L, A)=\frac{N_{C}}{\min \left(N_{P_{L}}, N_{P_{A}}^{C}\right)}
$$

Where notations have the same meaning as in Definition 3.2. If the class matching is accurate, $M(L, A)$ should be the same after applying class repackaging or when other classes are inside the same package. Similar to LiBID-S, we can use the Constraint 5 to decide if library $L$ is used in app $A$.

Summary. Overall, LiBID-A has enhanced the abilities of LIBID$\mathrm{S}$. By introducing finer-grained constraints, LIBID-A provides higher accuracy in terms of class-level matching, which further enables LIBID-A to pinpoint the version of libraries with greater accuracy. In addition, LIBID-A utilizes the dependency graphs to separate different library classes, which enables it to better deal with class repackaging and the interference of shared-package libraries. The adoption of finer-grained features will inevitably slow down the execution of LiBID-A. To achieve a balance between time efficiency and accuracy, LiBID-A also uses LSH Ensemble to speed up the matching process and apply various constraints to minimize false matches. The execution of LiBID-A for different apps is completely parallelizable.

\section{EVALUATION}

LIBID uses Androguard [3] version 2.0 with the DAD decompiler to construct the CFGs of methods. We extend the original Androguard project to support multi-dex files and our signature algorithms. In addition, we make use of the datasketch [41] package to implement the LSH Ensemble algorithm. All LSH parameters are set to default values that are tuned by benchmarks. The BIP models are implemented with the Gurobi [17] optimizer. We deploy LIBID on a computing cluster node with 32 Intel Xeon Gold 6142 CPUs; each CPU has 12040MB RAM.

To evaluate the effectiveness of LIBID, we collect 69 popular libraries with 1,444 versions from central repositories (e.g., Maven Central and JCenter), open-source platforms (e.g., Github), and 
official websites. Since Androguard cannot directly parse the downloaded library jars, we convert these . jar files to . dex files using the dex2jar [27] tool. The decompilation, however, does not work in all cases. During our experiment, around $94.17 \%$ of libraries are successfully converted, while LibScout successfully loads $94.47 \%$ libraries. In addition to LibScout, we compare LIBID with state-ofthe-art work, Orlis [35] and LibPecker [40], under different settings.

\subsection{Library Detection on Synthetic Apps}

To determine the detection threshold $\Gamma_{1}$ and $\Gamma_{2}$ in Constraint 5 , we propose a novel way to generate synthetic apps with different versions of libraries and different obfuscator configurations. We use ProGuard as the obfuscator due to its popularity. A recent study has shown that about $88 \%$ of obfuscated apps chose ProGuard as their only obfuscator, and $70 \%$ of them used the default ProGuard configuration [34].

Our method works by creating an Android project. Then, for each library in our dataset, we manually collect code snippets that provide main library functions from either the official documentation or example projects. We store these snippets in a database, along with the import statements, program, and dependencies for each library. In our dataset, the main APIs of most libraries are consistent for a wide range of versions. Therefore, a single generated app can be used with many library versions. We compile each app in the database under four different ProGuard settings, as shown in Table 2. Identifier renaming is enabled in all groups except when ProGuard is disabled.

We apply both tools to detect in-app libraries and calculate the number of true positives (TP), false positives (FP) and negatives (FN). Here, a false negative means the tool does not report any version of the library, while a false positive is counted if the tool reported some versions of the library excluding the correct one. Since we know in advance which library is used in each app, these evaluation indexes can be measured against ground truth. Then, we calculate the $F_{1}$ score to quantify the performance of both tools. To determine $\Gamma_{1}$ and $\Gamma_{2}$, we first set them to 1 and 0 , respectively, to observe the result and then gradually adjust them to appropriate values $\left(\Gamma_{1}=0.8, \Gamma_{2}=0.1\right)$ to achieve the highest $F_{1}$ score.

Evaluation. We tentatively compare the performance of LiBID with LibScout, Orlis, and LibPecker because they are state-of-the-art library detection tools which are designed to handle code shrinking. In particular, Orlis only reports matches between app and library classes. Therefore, we regard all library versions reported by Orlis as possible matches. For LibPecker, we set all thresholds based on the original paper. LibPecker reports the similarity between the app and each library candidate, and thus we choose the library version(s) with the highest similarity, if above the threshold, as the matched in-app library version(s).

Table 2 presents the experiment results under different ProGuard settings. It shows that LIBID achieves higher $F_{1}$ scores than other tools in all cases. When ProGuard is disabled, all tools except Orlis achieve high accuracy. Overall, Orlis is ineffective in identifying in-app library versions. Table 2 (b) shows that LIBID is much more effective than LibScout and LibPecker when class repackaging is enabled. LiBID accurately identifies libraries for more than $98.4 \%$ apps and reports fewer false negatives. In comparison, LibScout and
Table 2: Library Detection Results

(a) ProGuard Disabled

\begin{tabular}{cccccc}
\hline Tool & \# apps & \# TP & \# FP & \# FN & F $_{\mathbf{1}}$ \\
\hline Orlis & & 359 & 250 & 436 & 0.5114 \\
LibScout & & 969 & 33 & 43 & 0.9623 \\
LibPecker & 1,045 & 984 & 38 & 23 & 0.9699 \\
LiBID-S & & 1044 & 1 & 0 & 0.9995 \\
LiBID-A & & 1045 & 0 & 0 & 1 \\
\hline
\end{tabular}

(b) Class Repackaging Enabled

\begin{tabular}{cccccc}
\hline Tool & \# apps & \# TP & \# FP & \# FN & F $_{\mathbf{1}}$ \\
\hline Orlis & & 499 & 276 & 270 & 0.6464 \\
LibScout & & 7 & 2 & 1036 & 0.0133 \\
LibPecker & 1,045 & 100 & 66 & 879 & 0.1747 \\
LiBID-S & & 1029 & 5 & 11 & 0.9923 \\
LiBID-A & & 1028 & 14 & 3 & 0.9918 \\
\hline
\end{tabular}

(c) Shrinking Enabled (Default ProGuard Setting)

\begin{tabular}{cccccc}
\hline Tool & \# apps & \# TP & \# FP & \# FN & $\mathbf{F}_{\mathbf{1}}$ \\
\hline Orlis & & 226 & 169 & 537 & 0.3903 \\
LibScout & & 26 & 4 & 902 & 0.0543 \\
LibPecker & 932 & 126 & 90 & 716 & 0.2382 \\
LisID-S & & 924 & 8 & 0 & 0.9957 \\
LiBID-A & & 932 & 0 & 0 & 1 \\
\hline
\end{tabular}

(d) Shrinking and Class Repackaging Enabled

\begin{tabular}{cccccc}
\hline Tool & \# apps & \# TP & \# FP & \# FN & F $_{\mathbf{1}}$ \\
\hline Orlis & & 225 & 165 & 542 & 0.3889 \\
LibScout & & 0 & 0 & 932 & N/A \\
LibPecker & \multirow{2}{*}{932} & 22 & 47 & 863 & 0.0461 \\
LiBID-S & & 305 & 145 & 482 & 0.4931 \\
LiBID-A & & 831 & 40 & 61 & 0.9427 \\
\hline
\end{tabular}

LibPecker are correct for $0.7 \%$ and $9.6 \%$ apps, respectively, because they rely on the package hierarchy information.

Table 2 (c) demonstrates that other tools are also ineffective against code shrinking. In particular, if code shrinking is enabled, library code may be partially removed in the app. We define an index $I_{C}$ to quantify the library information remaining in the app:

$$
I_{C}=\frac{\text { \# Signatures of Library Classes in App }}{\text { \# Signatures of Library Classes in SDK }}
$$

Based on the definition, $I_{C}$ will be 1 if code shrinking is disabled. We calculate $I_{C}$ for every synthetic app that applied code shrinking. Because synthetic apps are relatively simple, most of them contain only a small portion of library classes after code shrinking. Fig. 5 presents the relation between the recall and $I_{C}$ when shrinking is enabled. It reveals that LIBID behaves pretty well against code shrinking, while LibScout, LibPecker and Orlis only detect libraries when $I_{C}>70 \%$, and still fail to detect many libraries in that case. 


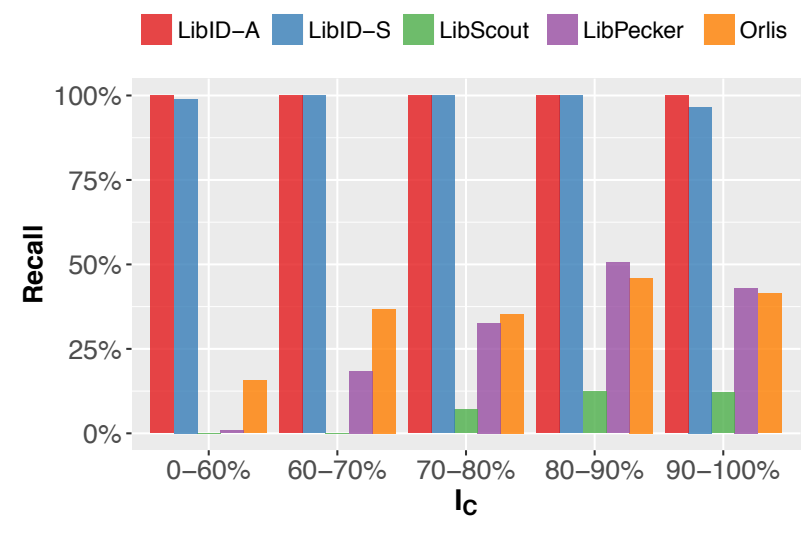

Figure 5: Recall under different shrinking range

The weakness of LiBID-S is clear when both shrinking and class repackaging are enabled. LiBID-S uses the library match index $M(L, A)$ in Definition 3.2 to quantify the confidence of a library $L$ being used in an app $A$. However, this value can be very low if the majority of library classes have been deleted (low $N_{C} / N_{P_{L}}$ ) while there are many other non-library classes in the same package (low $N_{C} / N_{P_{A}}$ ). By contrast, LiBID-A only considers app classes that are related to the library by eliminating other classes through ghost hunting and relation pruning, and thus its library match index in Equation 6 is still high for the right library. Nevertheless, LiBID-A does produce several false alarms. There are two reasons for the inaccuracy. First, class-level matching is not always accurate due to lack of hierarchy information, which would further influence the ghost hunting and relation pruning processes. Second, in a few cases, ProGuard keeps some library classes in the original package to ensure normal functioning while moving others to a single parent package, which could influence the accuracy of LiBID-A.

\subsection{Library Detection on F-Droid Apps}

Despite the promising results on synthetic apps, performance on more sophisticated apps may vary. In general, commercial apps may invoke more library functions than the collected code snippets, and they usually have a much larger code base and more complicated interactions between classes. In addition, using more advanced obfuscators may also affect the result. Therefore, we need a more realistic ground truth to evaluate the performance.

Wang et al. has compiled F-Droid projects under different obfuscators, including ProGuard, Allatori, and DashO, and published the dataset online [26]. The dataset contains 200-300 app binaries for each obfuscation configuration, with the ground truth of in-app library versions. However, apps in the dataset that should be obfuscated by ProGuard were, in fact, unobfuscated. Therefore, we manually compiled 215 F-Droid projects with Proguard applied (both code shrinking and class repackaging are enabled). We use both self-compiled apps and the dataset provided by Wang et al. as the ground truth to evaluate the performance of library detectors on commercial apps with different obfuscators. LiBID-S and LiBID-A are set with the thresholds determined in §5.1. Since all tools may report multiple library versions, we define two types of precision:
Table 3: Library Detection Results

(a) No Obfuscator

\begin{tabular}{cccc}
\hline Tool & Precision $^{*}$ & Recall & $\mathbf{F}_{\mathbf{1}}{ }^{*}$ \\
\hline Orlis & $49.82 \%(81.14 \%)$ & $26.25 \%$ & $0.3438(0.3966)$ \\
LibScout & $67.42 \%(69.35 \%)$ & $94.09 \%$ & $0.7855(0.7984)$ \\
LibPecker & $64.50 \%(65.26 \%)$ & $91.68 \%$ & $0.7573(0.7625)$ \\
LiBID-S & $67.42 \%(93.91 \%)$ & $88.35 \%$ & $0.7648(0.9105)$ \\
LiBID-A & $70.12 \%(88.16 \%)$ & $96.30 \%$ & $0.8115(0.9205)$ \\
\hline
\end{tabular}

(b) ProGuard (both shrinking and class repackaging enabled)

\begin{tabular}{cccc}
\hline Tool & Precision $^{*}$ & Recall & F $^{*}$ \\
\hline Orlis & $29.41 \%(62.50 \%)$ & $15.00 \%$ & $0.1987(0.2419)$ \\
LibScout & $65.22 \%(65.22 \%)$ & $15.00 \%$ & $0.2439(0.2439)$ \\
LibPecker & $20.00 \%(20.00 \%)$ & $4.00 \%$ & $0.0667(0.0667)$ \\
LiBID-S & $58.67 \%(66.67 \%)$ & $44.00 \%$ & $0.5029(0.5301)$ \\
LIBID-A & $64.29 \%(72.58 \%)$ & $45.00 \%$ & $0.5294(0.5556)$ \\
\hline
\end{tabular}

(c) Allatori

\begin{tabular}{cccc}
\hline Tool & Precision $^{*}$ & Recall & $\mathbf{F}_{\mathbf{1}}{ }^{*}$ \\
\hline Orlis & $46.46 \%(76.67 \%)$ & $19.37 \%$ & $0.2734(0.3092)$ \\
LibScout & $87.23 \%(91.11 \%)$ & $8.63 \%$ & $0.1571(0.1577)$ \\
LibPecker & $55.28 \%(65.26 \%)$ & $66.11 \%$ & $0.6021(0.6103)$ \\
LiBID-S & $73.72 \%(92.74 \%)$ & $48.42 \%$ & $0.5845(0.6362)$ \\
LiBID-A & $75.86 \%(92.22 \%)$ & $64.84 \%$ & $0.6992(0.7614)$ \\
\hline
\end{tabular}

(d) DashO

\begin{tabular}{cccc}
\hline Tool & Precision $^{*}$ & Recall & F $^{*}$ \\
\hline Orlis & $54.43 \%(87.76 \%)$ & $15.03 \%$ & $0.2356(0.2567)$ \\
LibScout & $90.64 \%(92.81 \%)$ & $54.20 \%$ & $0.6783(0.6843)$ \\
LibPecker & $47.06 \%(47.06 \%)$ & $55.94 \%$ & $0.5112(0.5112)$ \\
LiBID-S & $69.55 \%(97.45 \%)$ & $53.50 \%$ & $0.6047(0.6907)$ \\
LiBID-A & $69.79 \%(91.11 \%)$ & $57.34 \%$ & $0.6296(0.7039)$ \\
\hline
\end{tabular}

*: The value before the bracket is the pinpoint precision (or $F_{1}$ ); the value inside the brackets is the in-range precision (or $F_{1}$ ).

pinpoint precision and in-range precision. In particular, the pinpoint precision of a tool is the proportion of correctly identified libraries over all the libraries reported. When calculating the in-range precision, we regard all reported versions of a library as a single entity. If the reported versions of an entity include the correct version, then we refer to this entity as a correctly identified entity. The in-range precision of a tool is the proportion of correctly identified entities over all the entities reported. For example, if LiBID-A reports "ACRA-4.6.1, ACRA-4.6.2, Gson-2.5" while the ground truth is ACRA-4.6.1, then the respective pinpoint and in-range precision of LIBID-A are $1 / 3$ and $1 / 2$. The pinpoint and in-range $F_{1}$ scores are calculated using the pinpoint and in-range precision, respectively.

Table 3 presents the experiment results. Overall, LisID behaves better than state-of-the-art tools in all four cases. The $F_{1}$ score (both pinpoint and in-range) of LIBID is greater than 0.5 when ProGuard is applied, while that of other tools is lower than 0.25 . In 
Table 4: Top 5 libraries identified by LIBID

\begin{tabular}{cccc}
\hline Library & \# LiBID & \# LibScout & \# Common \\
\hline Gson & 1419 & 1114 & 1093 \\
Facebook & 1243 & 1082 & 1058 \\
Okhttp & 1157 & 690 & 689 \\
Okio & 1139 & 563 & 561 \\
Bolts & 918 & 317 & 316 \\
\hline
\end{tabular}

all cases, LIBID has the highest in-range precisions among these tools. Nevertheless, results also reveal that the performance of LIBID for these F-Droid apps is not as good as in §5.1. We investigate several cases where LiBID-A does not identify the library correctly and find the following causes: (i) $I_{C}$ is less than $5 \%$ for some libraries (e.g., Guava) after code shrinking, which is lower than the threshold; (ii) LiBID-A fails to identify some libraries when both shrinking and class repackaging are enabled for the same reason as in §5.1; and (iii) the obfuscation in some apps changes the class access flag and class interfaces and splits a single library class into several classes, which could defeat the design of LiBID-A.

\subsection{Libraries in Popular Google Play Apps}

To study the library usage in production apps and further evaluate our approach, we downloaded 3,958 apps from the top-100 apps across 59 categories on the Google Play store in April 2017. We employ both LiBID (using LiBID-A) and LibScout to analyze these popular apps. On average, LiBID detects one more library per app than LibScout. Table 4 gives the summary of top five most popular libraries identified by LIBID. In particular, the columns respectively present the number of apps using each library identified by LiBID, LibScout, and both of them. The table shows that a large proportion of libraries have been missed by LibScout. We confirm the reason is that LibScout struggles to detect libraries after code shrinking.

Vulnerable Library Detection. A primary use case of LiBID is to identify vulnerable in-app libraries. Here, we use OkHttp, a popular Android library used in $29 \%$ of apps in our dataset for managing HTTP-based network requests, as an example. There is a severe vulnerability in 0kHttp for versions $2 . x$ before 2.7.4, and 3.x before 3.1.2, that allows an attacker to bypass the certificate pinning (CVE-2016-2402) [8].

We apply LibID, LibScout, Orlis, and LibPecker to check if a vulnerable version of the OkHttp library is used in popular apps. The results are presented in Table 5. All tools find that many popular apps use at least one version of vulnerable OkHttp libraries. In particular, LiBID identifies 393 affected apps, while the figure for LibScout and LibPecker is 296 and 339, respectively. All tools but Orlis agree on 294 apps, but the remaining list of affected apps differs. We notice that OkHttp contains a field marking the actual version of the library, and this field has not been obfuscated in the majority of our test apps. Therefore, we only need a little manual effort to verify the results. Note that LIBID does not use string constants as a feature to detect libraries. Thus, the version information of OkHttp in the app binaries only helps us to confirm the result instead of assisting LIBID directly. For those apps that LIBID and LibScout disagree, all 99 additional apps reported by LiBID are true
Table 5: Vulnerable OkHttp library detection result

\begin{tabular}{ccccc}
\hline Tool & \# TP & \# FP & \# FN & F $_{\mathbf{1}}$ \\
\hline Orlis & 172 & 165 & 222 & 0.4706 \\
LibScout & 295 & 1 & 99 & 0.8551 \\
LibPecker & 339 & 9 & 55 & 0.9137 \\
LiBID & 393 & 0 & 1 & 0.9987 \\
\hline
\end{tabular}

positives. Meanwhile, LibScout uniquely reports 2 vulnerable apps, and one of them is a false positive; the other one is missed by LIBID. We further analyze the app that LiBID fails to detect. It turns out the app uses both a shrunk version of OkHttp 2.4.0 (vulnerable) and an unobfuscated version of OkHttp 3.4.1 (patched). During the matching process, LiBID mismatches the OkHttp 2.4.0 library to the OkHttp 3.4.1 package but the matching confidence does not reach the threshold. As a result, this vulnerable app is missed by LiBID.

Overall, the results demonstrate the accuracy and robustness of LIBID, but it also raises security concerns about the Android platform. Although the vulnerability of OkHttp was reported in February 2016, around $10 \%$ of popular apps in the Google Play dataset still use an insecure version of the OkHttp library. Fig. 7 in Appendix A presents the detailed distribution of vulnerable apps in our dataset. More than $30 \%$ of apps in two categories (NEWS_AND_MAGAZINES and TRAVEL_AND_LOCAL) and at least $10 \%$ of apps under 25 categories use vulnerable versions of OkHttp. Meanwhile, a large proportion of OkHttp libraries used in popular apps are unpatched, including more than $44 \%$ of OkHttp libraries in the top 100 free apps and the other six categories. We also discover that three of Google's apps use vulnerable versions of OkHttp libraries, all of which are installed by millions of users. Although we confirm that these three apps do not invoke the vulnerable certificate pinning method, it is still best practice to upgrade the library version to ensure vulnerable code is not introduced in a future release. Finally, we sent emails to the developer of the reported 393 vulnerable apps on December 23, 2017. As a result, we received 22 non-automatic replies. Among these replies, 17 said they will investigate, 3 have updated the $0 \mathrm{kHttp}$ library, and 2 promised to update it in the future release.

\subsection{Limitation}

LiBID selects several features to profile and detect libraries. These features, however, are not robust against more advanced obfuscation techniques. For instance, the class access flag and class interfaces can be modified if "allowaccessmodification" and "mergeinterfacesaggressively" are enabled in ProGuard. API hiding obfuscation can also hide calls to the Android SDK through Java reflection. Nevertheless, these cases are relatively rare compared with code shrinking and package modification operations (§5.2). In addition, ProGuard suggests users exploit these advanced features cautiously because they can reduce the performance and may cause JVM problems [16]. The aim of LIBID is to be the first to reliably detect libraries under the most popular obfuscation functions, including code shrinking, identifier renaming, control flow randomization, and package modifications. We achieve this goal.

One major concern of LIBID is the scalability. Using library detection on unobfuscated F-Droid apps as an example (§5.2), we 
Table 6: Average Time Consumption

\begin{tabular}{cccc}
\hline Tool & Library Profiling & App Profiling & Matching \\
\hline Orlis & $5.18 \mathrm{~s}$ & - & $850.30 \mathrm{~s}$ \\
LibScout & $1.01 \mathrm{~s}$ & - & $1.63 \mathrm{~s}$ \\
LibPecker & - & - & $509.40 \mathrm{~s}$ \\
LiBID-S & $4.13 \mathrm{~s}$ & $38.36 \mathrm{~s}$ & $2.26 \mathrm{~s}$ \\
LiBID-A & $4.13 \mathrm{~s}$ & $38.36 \mathrm{~s}$ & $11.25 \mathrm{~s}$ \\
\hline
\end{tabular}

calculate the average time spent on profiling a library, profiling an app, and matching an app with libraries for each tool. The results are presented in Table 6. Although LiBID is more time efficient than Orlis and LibPecker, it spent more time than LibScout. This is because LiBID performs a finer-grained analysis compared with LibScout. Both the CFG construction and candidate class matching are time-consuming. The BIP solver could also take a long time to find a solution if there are too many constraints. Nevertheless, the use of LSH Ensemble has greatly improved the matching time by avoiding pair-wise comparison and we design two working schemes to prioritize either scalability or accuracy. As seen in Table 6, LiBID-S spent much less time in the matching process because it does not make use of the dependency graph. In addition, for large-scale analysis, we can run LIBID in parallel on a computing cluster since each task is independent. In general, LiBID-A is preferred for small-scale analysis, or large-scale analysis when there are enough computing resources; LIBID-S is favored in other cases.

\section{RELATED WORK}

The high prevalence of third-party libraries in mobile apps has been an obstacle to app clone detection research for many years. Most of the existing studies employed naive whitelisting techniques to identify and exclude common libraries before analysis, either by comparing package names $[1,9]$ or the hash value of known libraries [10]. However, these approaches can miss many less-popular libraries, and the lack of granularity makes them incapable of detecting obfuscated libraries. More advanced work filters libraries by clustering. WuKong [33] chose the frequency of Android API calls as the feature to identify libraries by clustering. It reported that third-party libraries contributed more than $60 \%$ of the code in Android apps. Andarwin [11] grouped similar semantic blocks from the Program Dependence Graph (PDG) to detect library and applied LSH techniques to accelerate the clustering process. LibDetect [14] identified and removed in-app library classes from an app using fuzzy hashing to increase the accuracy of app clone detection.

Grace et al. and Book et al. studied the presence and behavior of advertising libraries using a whitelist $[7,15]$. Li et al. harvested 1,113 common libraries from 1.5 million Google Play apps by comparing the name of in-app methods and packages [18]. LibRadar [21] implemented and improved WuKong by providing an online detection platform and designing a better cluster algorithm. LibD [19] detected and classified library candidates based on dependencies between methods and packages. LibSift [29] performed library detection by comparing the primary components of the PDG. In addition, machine learning techniques have been applied to detect third-party libraries [20,22]. Nevertheless, these proposals rely on the package hierarchies and can neither pinpoint library version nor handle popular obfuscation techniques such as code shrinking.

MobScanner [6] used weighted features to identify in-app library versions. However, it relies on string constants and is unreliable when intensive code shrinking is applied. Similar to LIBID, LibScout [5] built library profiles from pre-collected library binaries. LibScout used a fuzzy descriptor to generate a method signature, and further obtain a class signature using the hash of in-class methods. Then, LibScout calculates the similarity of class signatures between a candidate package and a known library package to determine whether they are a match. Although LibScout is resilient against identifier renaming operations, it performs poorly if either dead-code elimination or class repackaging is enabled. OSSPolice [12] was designed to identify license violations of third-party Open Source Software (OSS) in mobile apps. It extracted more features (e.g., string constants) from library binaries to better pinpoint library versions. Nevertheless, OSSPolice is only resilient to simple obfuscation techniques such as identifier renaming. Ordol [31] is another tool that can detect the library version in Android binaries. It assigned a weight to every method and class based on the number of instructions and tried to find the maximum weight bipartite matchings. Although it does not rely on any hierarchy information, Ordol requires pair-wise comparison and its threat model does not include code shrinking and package modifications.

Recently, LibPecker [40] utilized the class dependencies to perform obfuscation-resilient library matchings. Instead of matching the dependency graph, they encode the graph into a set of fuzzy class signatures and calculate the Jaccard similarity between the library and app signatures to decide whether two classes are a match. Although the class dependencies provide additional entropy in the class signature, LibPecker relies on the package hierarchy and cannot handle drastic code shrinking. Orlis [35] was also designed to be resilient to code shrinking and class repackaging. The authors have shown that Orlis outperformed LibDetect when identifying obfuscated in-app library classes. However, as shown in $\S 5$, Orlis is not designed to detect specific library versions.

\section{CONCLUSION}

This paper has presented LIBID, a reliable tool that identifies thirdparty Android libraries and their version in app binaries. LIBID includes two detection schemes: LiBID-S and LiBID-A, which focus on scalability and accuracy, respectively. We convert the abstract library identification problem into a BIP problem that has been well studied in the research community. Overall, LiBID overcomes several limitations found in previous work and is able to determine the version of third-party libraries used in an app binary using identifier renaming, shrinking, control flow randomization, and package modification techniques. Evaluation is supported by our novel method that semi-automatically generates apps containing third-party libraries, as well as an analysis of hundreds of F-Droid apps. This provides valuable ground truth data to support accurate evaluation and comparison of our approach to previous work. Our experiments show that LIBID can detect many more libraries than prior art, especially when code shrinking is enabled and the package hierarchy is modified. LIBID is open source software and we encourage researchers to use this tool to assist their own research. 


\section{ACKNOWLEDGMENTS}

Jiexin Zhang is supported by the China Scholarship Council. Alastair R. Beresford is partly supported by The Boeing Company and EPSRC under Grant No.: EP/M020320/1. Stephan A. Kollmann is supported by Microsoft Research and The Boeing Company. The opinions, findings, and conclusions or recommendations expressed are those of the authors and do not necessarily reflect those of the funders. We thank Diana A. Vasile, Ricardo Mendes, Martin Kleppmann, and Peidong Zhu for helpful discussion and insight. We also thank anonymous reviewers for their feedback on the paper.

\section{REFERENCES}

[1] Yousra Aafer, Wenliang Du, and Heng Yin. 2013. Droidapiminer: Mining ApiLevel Features for Robust Malware Detection in Android. In International Conference on Security and Privacy in Communication Systems. Springer, 86-103.

[2] Alexandr Andoni and Piotr Indyk. 2006. Near-Optimal Hashing Algorithms for Approximate Nearest Neighbor in High Dimensions. In Foundations of Computer Science, 2006. FOCS'06. 47th Annual IEEE Symposium on. IEEE, 459-468.

[3] Androguard. 2018. Androguard: Reverse Engineering, Malware and Goodware Analysis of Android Applications. (Jan. 2018). Retrieved Janurary, 2019 from https://github.com/androguard/androguard

[4] Vitalii Avdiienko, Konstantin Kuznetsov, Alessandra Gorla, Andreas Zeller, Steven Arzt, Siegfried Rasthofer, and Eric Bodden. 2015. Mining Apps for Abnormal Usage of Sensitive Data. In Proceedings of the 37th International Conference on Software Engineering-Volume 1. IEEE Press, 426-436.

[5] Michael Backes, Sven Bugiel, and Erik Derr. 2016. Reliable Third-Party Library Detection in Android and Its Security Applications. In Proceedings of the 2016 ACM SIGSAC Conference on Computer and Communications Security. ACM, 356-367.

[6] Salman A Baset, Shih-Wei Li, Philippe Suter, and Omer Tripp. 2017. Identifying Android Library Dependencies in the Presence of Code Obfuscation and Minimization. In 2017 IEEE/ACM 39th International Conference on Software Engineering Companion (ICSE-C). IEEE, 250-252.

[7] Theodore Book, Adam Pridgen, and Dan S Wallach. 2013. Longitudinal Analysis of Android Ad Library Permissions. arXiv preprint arXiv:1303.0857 (2013).

[8] Computer Security Resource Center. 2016. CVE-2016-2402 Detail. (Jan. 2016) Retrieved Janurary, 2019 from https://nvd.nist.gov/vuln/detail/CVE-2016-2402

[9] Kai Chen, Peng Liu, and Yingjun Zhang. 2014. Achieving Accuracy and Scalability Simultaneously in Detecting Application Clones on Android Markets. In Proceedings of the 36th International Conference on Software Engineering. ACM, 175-186.

[10] Jonathan Crussell, Clint Gibler, and Hao Chen. 2012. Attack of the Clones: Detecting Cloned Applications on Android Markets. In ESORICS, Vol. 12. Springer, 37-54.

[11] Jonathan Crussell, Clint Gibler, and Hao Chen. 2015. Andarwin: Scalable Detection of Android Application Clones Based on Semantics. IEEE Transactions on Mobile Computing 14, 10 (2015), 2007-2019.

[12] Ruian Duan, Ashish Bijlani, Meng Xu, Taesoo Kim, and Wenke Lee. 2017. Identifying Open-Source License Violation and 1-Day Security Risk at Large Scale. In Proceedings of the 2017 ACM SIGSAC Conference on Computer and Communications Security. ACM, 2169-2185.

[13] Felix Fischer, Konstantin Böttinger, Huang Xiao, Christian Stransky, Yasemin Acar, Michael Backes, and Sascha Fahl. 2017. Stack Overflow Considered Harmful? The Impact of Copy\&Paste on Android Application Security. In Symposium on Security and Privacy (Oakland'17). IEEE.

[14] Leonid Glanz, Sven Amann, Michael Eichberg, Michael Reif, Ben Hermann, Johannes Lerch, and Mira Mezini. 2017. CodeMatch: Obfuscation Won't Conceal Your Repackaged App. In Proceedings of the 2017 11th foint Meeting on Foundations of Software Engineering. ACM, 638-648.

[15] Michael C Grace, Wu Zhou, Xuxian Jiang, and Ahmad-Reza Sadeghi. 2012. Unsafe Exposure Analysis of Mobile In-App Advertisements. In Proceedings of the fifth ACM conference on Security and Privacy in Wireless and Mobile Networks. ACM, 101-112.

[16] GuardSquare. 2019. ProGuard Manual. (Jan. 2019). Retrieved Janurary, 2019 from https://www.guardsquare.com/en/proguard/manual/introduction

[17] Inc. Gurobi Optimization. 2016. Gurobi Optimizer Reference Manual. (March 2016). Retrieved March, 2018 from http://www.gurobi.com

[18] Li Li, Tegawendé F Bissyandé, Jacques Klein, and Yves Le Traon. 2016. An Investigation into the Use of Common Libraries in Android Apps. In Software Analysis, Evolution, and Reengineering (SANER), 2016 IEEE 23rd International Conference on, Vol. 1. IEEE, 403-414.

[19] Menghao Li, Wei Wang, Pei Wang, Shuai Wang, Dinghao Wu, Jian Liu, Rui Xue, and Wei Huo. 2017. Libd: Scalable and Precise Third-Party Library Detection in Android Markets. In Proc. 39th Int. Conf. Softw. Eng.(ICSE).
[20] Bin Liu, Bin Liu, Hongxia Jin, and Ramesh Govindan. 2015. Efficient Privilege De-Escalation for Ad Libraries in Mobile Apps. In Proceedings of the 13th Annual International Conference on Mobile Systems, Applications, and Services. ACM, 89-103.

[21] Ziang Ma, Haoyu Wang, Yao Guo, and Xiangqun Chen. 2016. Libradar: Fast and Accurate Detection of Third-Party Libraries in Android Apps. In Proceedings of the 38th International Conference on Software Engineering Companion. ACM, 653-656.

[22] Annamalai Narayanan, Lihui Chen, and Chee Keong Chan. 2014. Addetect: Automated Detection of Android Ad Libraries using Semantic Analysis. In Intelligent Sensors, Sensor Networks and Information Processing (ISSNIP), 2014 IEEE Ninth International Conference on. IEEE, 1-6.

[23] The Hacker News. 2014. Facebook SDK Vulnerability Puts Millions of Smartphone Users' Accounts at Risk. (Jan. 2014). Retrieved Janurary, 2019 from http:// thehackernews.com/2014/07/facebook-sdk-vulnerability-puts.html

[24] The Hacker News. 2015. Backdoor in Baidu Android SDK Puts 100 Million Devices at Risk. (Jan. 2015). Retrieved Janurary, 2019 from https://thehackernews.com/ 2015/11/android-malware-backdoor.html

[25] The Hacker News. 2015. Warning: 18,000 Android Apps Contains Code that Spy on Your Text Messages. (Jan. 2015). Retrieved Janurary, 2019 from http: //thehackernews.com/2015/11/android-malware-backdoor.html

[26] presto osu. 2019. Orlis/Orcis. (Jan. 2019). Retrieved Janurary, 2019 from https://github.com/presto-osu/orlis-orcis

[27] pxb1988. 2019. Dex2jar: Tools to Work with Android .dex and Java .class Files. (Jan. 2019). Retrieved Janurary, 2019 from https://github.com/pxb1988/dex2jar

[28] Shashi Shekhar, Michael Dietz, and Dan S Wallach. 2012. AdSplit: Separating Smartphone Advertising from Applications. In USENIX Security Symposium, Vol. 2012.

[29] Charlie Soh, Hee Beng Kuan Tan, Yauhen Leanidavich Arnatovich, Annamalai Narayanan, and Lipo Wang. 2016. LibSift: Automated Detection of Third-Party Libraries in Android Applications. In Software Engineering Conference (APSEC), 2016 23rd Asia-Pacific. IEEE, 41-48.

[30] Hamdy A Taha. 2014. Integer Programming: Theory, Applications, and Computations. Academic Press.

[31] Dennis Titze, Michael Lux, and Julian Schuette. 2017. Ordol: Obfuscation-Resilient Detection of Libraries in Android Applications. In Trustcom/BigDataSE/ICESS, 2017 IEEE. IEEE, 618-625.

[32] IDC Corporate USA. 2018. Smartphone Shipments Expected to Further Decline in 2018 Before Returning to Growth in 2019. (Jan. 2018). Retrieved Janurary, 2019 from https://www.idc.com/getdoc.jsp?containerId=prUS44529618

[33] Haoyu Wang, Yao Guo, Ziang Ma, and Xiangqun Chen. 2015. Wukong: A Scalable and Accurate Two-Phase Approach to Android App Clone Detection. In Proceedings of the 2015 International Symposium on Software Testing and Analysis. ACM, 71-82.

[34] Yan Wang and Atanas Rountev. 2017. Who Changed You? Obfuscator Identification for Android. In Proceedings of the 4th International Conference on Mobile Software Engineering and Systems. IEEE Press, 154-164.

[35] Yan Wang, Haowei Wu, Hailong Zhang, and Atanas Rountev. 2018. Orlis: Obfuscation-Resilient Library Detection for Android. In Proceedings of the 5th International Conference on Mobile Software Engineering and Systems (MOBILESoft '18). ACM, New York, NY, USA, 13-23.

[36] Takuya Watanabe, Mitsuaki Akiyama, Fumihiro Kanei, Eitaro Shioji, Yuta Takata, Bo Sun, Yuta Ishi, Toshiki Shibahara, Takeshi Yagi, and Tatsuya Mori. 2017. Understanding the Origins of Mobile App Vulnerabilities: A Large-scale Measurement Study of Free and Paid Apps. In Proceedings of the 14th International Conference on Mining Software Repositories. IEEE, 14-24.

[37] Fengguo Wei, Sankardas Roy, Xinming Ou, et al. 2014. Amandroid: A Precise and General Inter-Component Data Flow Analysis Framework for Security Vetting of Android Apps. In Proceedings of the 2014 ACM SIGSAC Conference on Computer and Communications Security. ACM, 1329-1341.

[38] Jesse Wilson. 2016. OkHttp Certificate Pinning Vulnerability. (Jan. 2016). Retrieved Janurary, 2019 from https://publicobject.com/2016/02/11/okhttpcertificate-pinning-vulnerability/

[39] Wenbo Yang, Juanru Li, Yuanyuan Zhang, Yong Li, Junliang Shu, and Dawu Gu. 2014. APKLancet: Tumor Payload Diagnosis and Purification for Android Applications. In Proceedings of the 9th ACM symposium on Information, computer and communications security. ACM, 483-494.

[40] Yuan Zhang, Jiarun Dai, Xiaohan Zhang, Sirong Huang, Zhemin Yang, Min Yang, and Hao Chen. 2018. Detecting Third-Party Libraries in Android Applications with High Precision and Recall. In Proceedings of the 2018 IEEE 25th International Conference on Software Analysis, Evolution and Reengineering (SANER). IEEE, 141-152.

[41] Eric Zhu. 2019. Datasketch: Big Data Looks Small. (Jan. 2019). Retrieved Janurary, 2019 from https://github.com/ekzhu/datasketch

[42] Erkang Zhu, Fatemeh Nargesian, Ken Q Pu, and Renée J Miller. 2016. LSH Ensemble: Internet-scale Domain Search. Proceedings of the VLDB Endowment 9, 12 (2016), 1185-1196. 


\section{A SUPPORTING TABLES AND FIGURES}

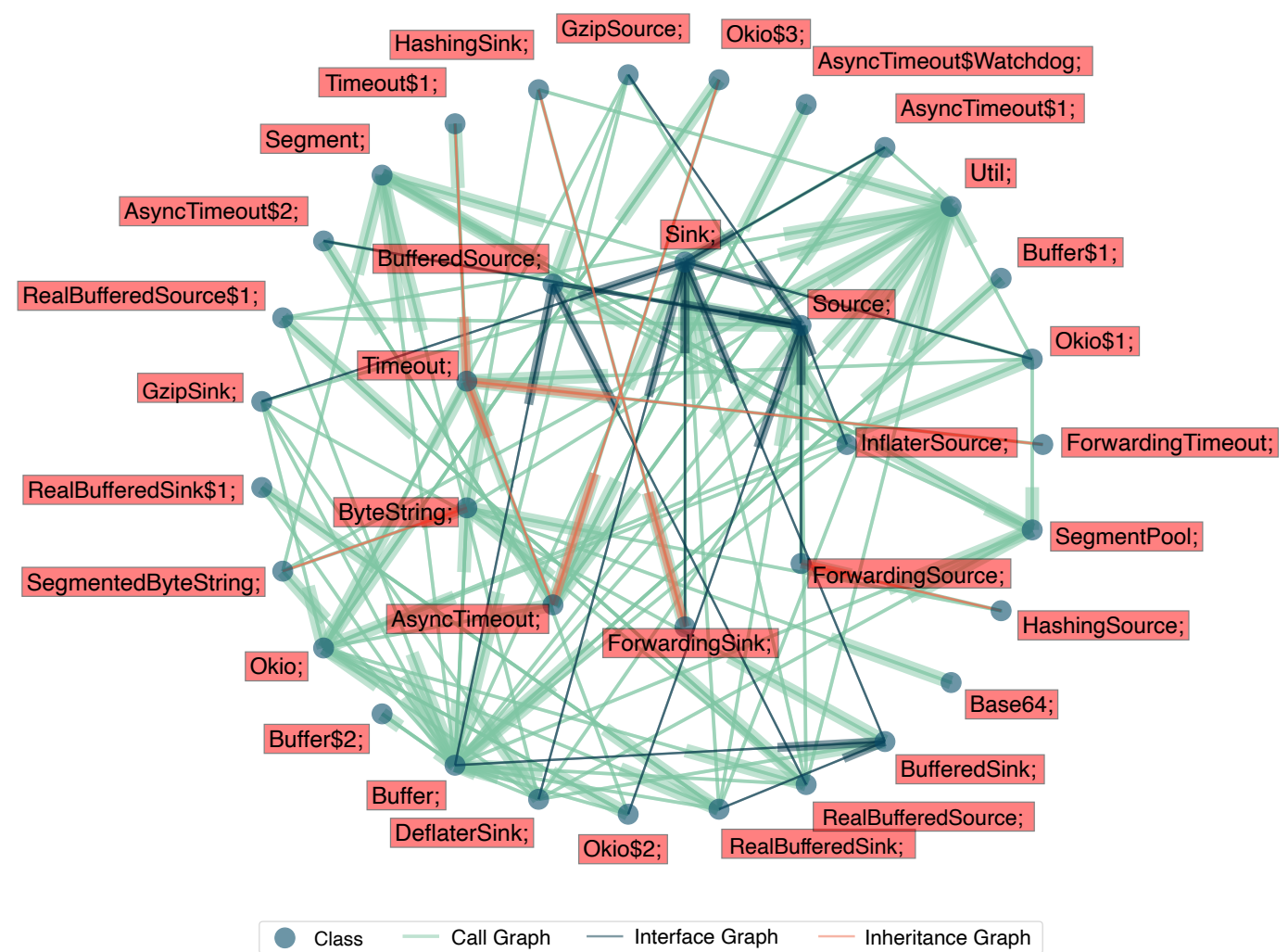

Figure 6: Dependency graph of Okio 1.7.0 (direction is represented by the thick line)
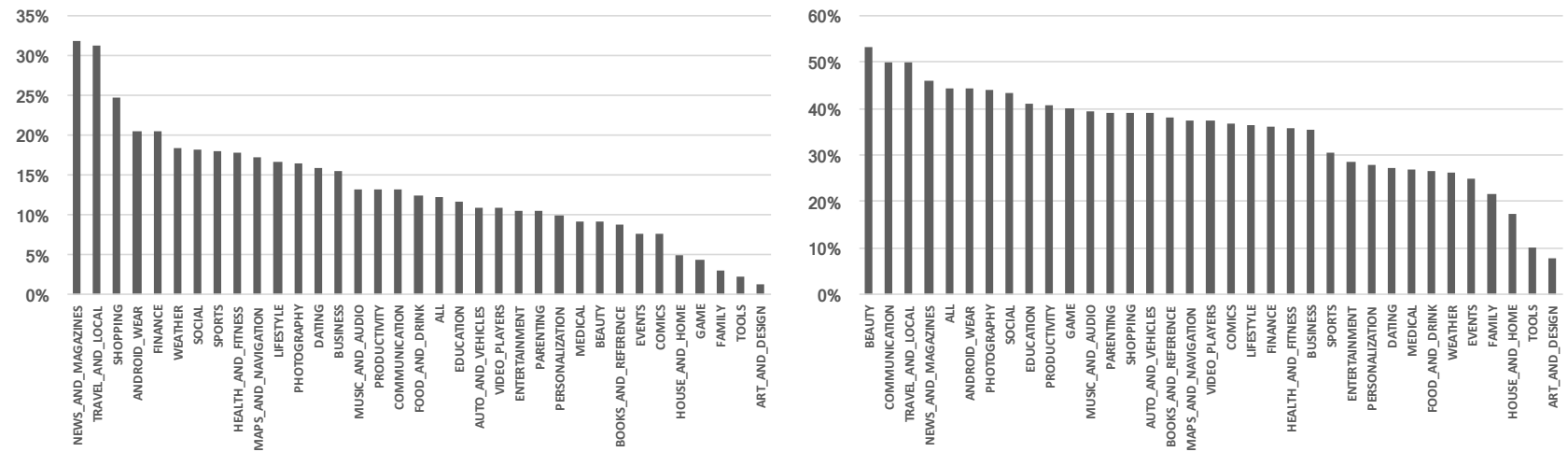

Figure 7: The ratio of apps using vulnerable OkHttp to apps under each category (left) and to apps using OkHttp under each category (right) 